\title{
Investigating the effects of bistatic SAR phenomenology on feature extraction
}

\author{
Michael Woollard, Matthew Ritchie, Hugh Griffiths \\ Electronic and Electrical Engineering Department \\ University College London \\ London, WC1E 7JE \\ Email: \{michael.woollard.15,m.ritchie,h.griffiths\}@ucl.ac.uk
}

\begin{abstract}
Interest in bistatic radar has fluctuated since its first demonstration. Modern multistatic and MIMO radar systems have prompted a resurgence in the field, particularly where imaging radar and automatic target recognition are concerned. The lack of openly-available bistatic imagery and corresponding analysis of the unique artefacts which occur within it is a significant barrier to developing automatic target recognition methods for such systems. This paper looks to address these issues by presenting an appropriate simulation methodology for obtaining bistatic synthetic aperture radar imagery of ground vehicle targets and investigating the features that occur within this imagery. In this paper, a number of effects unique to the bistatic case are presented, and the performance degradation of a classifier at several bistatic angles is empirically demonstrated. A version of the final database will be publicly released to promote wider research into this challenge.
\end{abstract}

\section{INTRODUCTION}

Synthetic Aperture Radar (SAR) has been a revolutionary sensing modality since its inception in the early 1950s. A review of early developments in SAR [1] describes some of the first openly published results using this technology. Since these early measurements, SAR has become a cornerstone of modern intelligence, surveillance, target acquisition, and reconnaissance (ISTAR) capabilities, due to its ability to sense over long ranges, by day or night, with little or no degradation by weather. Appropriate choice of operating frequency can also allow penetration of foliage and cover, whilst coherent change detection and interferometric modes allow precise detection and monitoring of changes in a scene which are undetectable by optical sensors.

The future of radar sensing is moving towards networked solutions containing multiple agile RF sensor nodes, due to the many advantages this brings. This approach realises a platform through which the recently developed ideas of cognition for radar sensing can be best exploited. As progressively greater information regarding the background scene or targets is obtained, the cognitive feedback loop provides the opportunity to change geometry, waveform or other variables under control of the wider system in order to improve the overall detection or classification probability. Little attention has been paid to the additional complexity introduced by variable bistatic collection geometries. It is therefore important to ensure that the problem of automatic target recognition (ATR) implementation is studied with the same rigour as was previously applied to the monostatic case.

Monostatic SAR ATR is a mature area of research and has benefited from the release of real databases such as MSTAR [2]. When simulating simulate or generate experimental multistatic SAR imagery, caution must be exercised when transferring across methods developed for monostatic SAR. It is widely accepted that such methods are not applicable to bistatic scenarios that cannot be approximated as quasimonostatic. For example, it is shown in [3] that bistatic SAR images of vehicles cannot be accurately co-registered to equivalent monostatic images for bistatic collection angles greater than $4^{\circ}$. More importantly, it is noted that accurate coregistration was only achievable for configurations in which the bistatic and monostatic apertures overlapped. This would imply data incest in a large-scale data fusion system such as a distributed MIMO radar.

This paper aims to address this gap by developing a comprehensive understanding of the effects introduced by the bistatic scattering configuration, and thus to better inform bistatic ATR processing methods. Section II defines the context of this research. Section III outlines the simulation methodology adopted and the approach to analysis. Section IV shows representative results from the work performed to date. Further work and conclusions are addressed in Section V.

\section{Problem CONTEXT}

\section{A. Simulation of bistatic SAR features}

Geometrical optics and the geometrical theory of diffraction (GTD) method [4] have been applied to this problem in order to develop canonical scattering models for simple targets [5][6][7][8]. Most of these models were verified against the XPATCH simulator [9][10], which was publicly released by the US Department of Defense in 1997. However, it is difficult to build complex targets from these canonical features when the effects of multipath returns must be considered. The simulated returned power for many vehicular targets is typically 10 to $15 \mathrm{~dB}$ lower if multipath is not considered [9]. XPATCH addressed the multipath problem by applying a shooting and bouncing ray (SBR) technique; this is commonly referred to as ray-tracing. This allows the use of CAD models for the desired ground targets to produce high fidelity simulated SAR imagery. 
The simulation work completed within this paper applies the recently released RaySAR [11] model, another SBR approach that was released to the wider research community in Jan 2016 and offering an open-source alternative to XPATCH. The ray-tracing methodology used by RaySAR was presented in [12]. A high-throughput pipeline based on the RaySAR simulator has previously been demonstrated [13], allowing the generation of large databases of sample imagery along with the associated ray-tracing contributions for further analysis. This approach has previously been applied to vehicular targets in order to demonstrate low-latency ATR methods. The same pipeline is used in this work to enable rapid analysis of complex targets and identify target poses or collection geometries which are particularly challenging for feature extraction.

Dstl recently released another SAR simulator called SARCASTIC [14][15]. This tool allows complex phase history data to be simulated, leading to full complex output images, and can account for material properties such as surface roughness to add realistic speckle to the final imagery. A direct comparison of the SARCASTIC simulation output with equivalent RaySAR configurations is shown in Subsection IV-A.

\section{B. SAR ATR}

The US Air Force Research Laboratories (AFRL) Moving and Stationary Target Acquisition and Recognition (MSTAR) database [2] has been used as the de facto standard for benchmarking ATR performance on SAR imagery for the last 20 years. The data was captured with a STARLOS system during 1995 and 1996, with a nominal resolution of 1 foot in both range and azimuth. The database includes ten targets, primarily military vehicles of Russian origin, along with samples of clutters and confuser targets.

There are several issues, however, with the MSTAR dataset which render it unsuitable for current ATR research. Firstly, the STARLOS system used to collect the data is not representative of modern SAR capabilities. Since the release of MSTAR, significant progress has been made in the area of ultra-wideband SAR systems leading to increases in both image resolution and quality. Figure 1 shows an image collected by the Bright Spark demonstrator system (a joint Dstl-Thales UK project) with clear shadow edges and high-resolution detail of the cars in the image ${ }^{1}$. The achieved resolution is $4 \mathrm{~cm}$ in range and $2.8 \mathrm{~cm}$ in azimuth, which is an order of magnitude improvement over STARLOS. Secondly, the scenes used to generate the MSTAR challenge often contain common clutter within a target class; this has been shown to artificially inflate the scores achieved by many CNN methods on the MSTAR challenge [16]. Finally, the entire database was collected in a monostatic configuration; as already mentioned in Section I, this makes the data unsuitable for developing bistatic ATR methods.

Recent approaches to the MSTAR classification challenge have heavily favoured neural network models. In particular,

\footnotetext{
${ }^{1}$ Publicly released imagery from Bright Spark is available at https://dstl.github.io/brightspark-data/data/
}

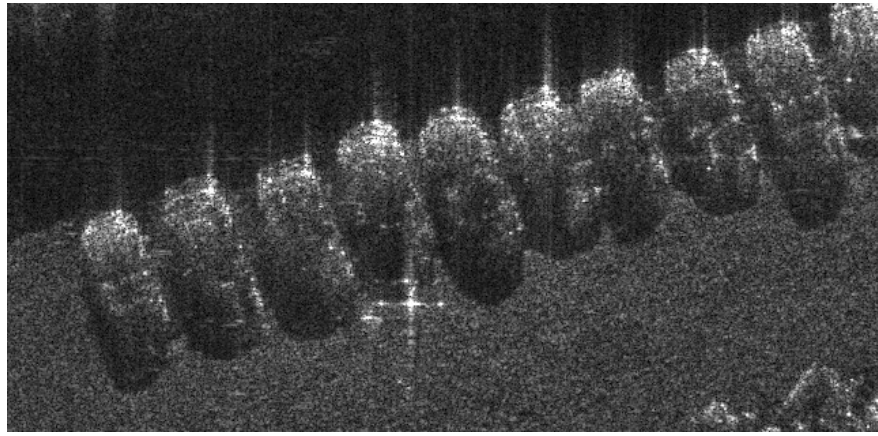

Fig. 1: A Bright Spark image capture showing several cars in a car park, with clearly visible shadows

convolutional neural networks (CNNs) have been applied to achieve very high accuracy scores. This parallels similar advances in the optical object detection and classification domains. High performance metrics, however, do not replace the need for explainability [17]. Whilst methods for producing human-interpretable explanations for neural network outputs, such as Grad-CAM [18] and saliency mapping [19], have made significant progress in recent years, the outputs are still difficult to interpret for a layman and do not offer a pointby-point justification for a given classification decision. For this reason, high-risk decisions such as targeting for kinetic operations are typically taken on the basis of deterministic methods such as template matching. At the bare minimum, a physical explanation for the presence of a feature in the image must be available before it can be used in targeting.

\section{Bistatic features and shadow region exploitation}

It has previously been observed that many man-made targets exhibit aspect-dependant scattering behaviour, which can be exploited to separate them from natural clutter [20]. Bistatic systems enable greater exploitation of this phenomenon, particularly where targets have been designed for low radar signature. It must be remembered, however, that the support in the wavenumber domain and hence the achievable resolution of a SAR system decreases with increasing bistatic angle. This can be derived for the zero grazing angle case with a stationary transmitter from Equation 1 as shown in [21]; $\Delta \theta$ is the accumulative angle across the aperture, $2 \theta_{b c}$ is the bistatic angle at the centre of the aperture, the radar waveform varies in frequency from $f_{c}$ to $f_{c}+B$ and $S$ is the total area of the support manifold.

$$
S=\frac{4 \pi^{2}}{c^{2}}\left[\Delta \theta+2 \sin \frac{\Delta \theta}{2} \cos \left(2 \theta_{b c}\right)\right]\left(f_{c}+\frac{B}{2}\right) B
$$

Consider a monostatic SAR transceiver imaging a scene. There may be areas with no direct (single-bounce) propagation path to the transceiver at any point along the aperture. This leads to the presence of a shadow in the reconstructed image, henceforth referred to as the reconstruction shadow. In the bistatic case, a second shadow is apparent due to the target being illuminated along a vector which is not equivalent to 

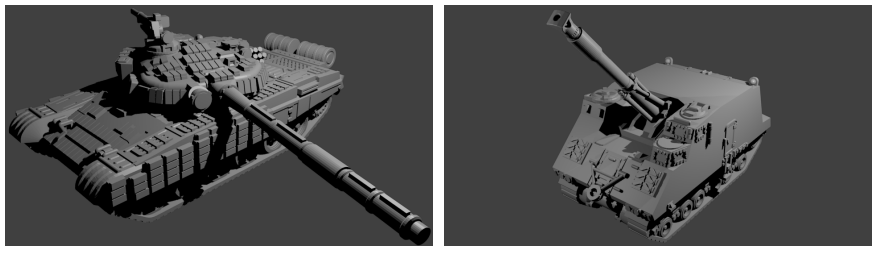

Fig. 2: Renders of the CAD models used for the T72B and L33 targets

that along which the receiver looks at the target. This will be referred to as the illumination shadow.

There is an important distinction to be made regarding this scenario, which is that a propagation path is not equivalent to a line-of-sight. Atmospheric interactions such as ducting or target interactions which are not a direct skin return can allow propagation along non-optical paths. In this work, it has been assumed that microwave radars emitting sufficiently high frequencies are used to achieve the resolutions discussed, and that the assumption of optical equivalence holds. Lowfrequency systems would not be able to make this assumption.

Prior work on shadow region features [16] used the SARBake tool [22] to segment the MSTAR images for analysis. This raises the question of whether it is possible to reliably segment shadows within an actual SAR image via edge detection techniques, for example, without prior knowledge of the targets in the scene. Use of information in the shadow regions as features for classification has previously been demonstrated [23][24].

\section{METHODS}

Using the simulation pipeline shown in [13], a large database of simulated imagery has been constructed covering five military targets and two civilian pickup truck variants ${ }^{2}$. Figure 2 shows two of the CAD models used for the simulations; representative SAR images formed by this process for monostatic and bistatic cases are shown in Figure 3. A resolution of $15 \mathrm{~cm}$ has been used in both range and azimuth; the pixel spacing in the images shown here is $5 \mathrm{~cm}$ unless stated otherwise. A limitation of the current configuration of the RaySAR implementation is that it does not account for surface roughness; therefore, the images are free from speckle contributions which would typically be observed within real SAR imagery.

Imagery was generated for each target as follows. The 3D CAD model of each target was placed on a ground plane at the origin. The transmitter and receiver nodes were placed such that they were located on the surface of a $500 \mathrm{~m}$ sphere centred on the origin and lay in the same horizontal plane. The elevation angle $\theta$ was varied in $5^{\circ}$ increments from $30^{\circ}$ to $60^{\circ}$, and the azimuth angle $\phi$ of the transmitter node was varied in $1^{\circ}$ increments from $0^{\circ}$ to $359^{\circ}$. For the monostatic case,

\footnotetext{
${ }^{2}$ Military targets from Marco Bergman's 1:100 scale tank library (https://www.thingiverse.com/thing:769137). Pickup truck models from https://free3d.com/3d-model/chevrolet-silverado-1986-jeep-44547.html and https://free3d.com/3d-model/pickup-69582.html
}

TABLE I: Key parameters for SARCASTIC configuration

\begin{tabular}{c|c} 
Parameter & Value \\
\hline Slant range to SRP & $5 \mathrm{~km}$ \\
Centre frequency & $10 \mathrm{GHz}$ \\
Bandwidth & $1413.235 \mathrm{MHz}$ \\
Aperture length & $719.5 \mathrm{~m}$ \\
Platform velocity & $50 \mathrm{~m} \mathrm{~s}^{-1}$ \\
Pulse repetition frequency & $2000 \mathrm{~Hz}$ \\
Pulse duration & $20 \mathrm{~ns}$
\end{tabular}

the transmitter and receiver were co-located (i.e. the bistatic angle $\beta=0$; for the bistatic cases, the receiver was offset by an angle $\beta$ from the transmitter in azimuth, where $\beta \in$ $\{-135,-90,-45,-20,-10,-5,5,10,20,45,90,135,180\}$ degrees.

A total of 35280 configurations were used per target (360 azimuth angles, 7 elevation angles, 14 bistatic angles). Where models could be posed, such as through the rotation of turrets or elevation of barrels, a number of poses were used; it should be noted that no analysis has been performed on the relative classification success for the same target model in different poses (this will from the basis of future investigations). The dataset was then divided to form segregated training and test sets; $30 \%$ of the images were selected at random to form the test set, and the remaining images constituted the training set.

The end goal of this analysis is to identify features which explain how classification success varies as a function of target type and collection geometry. A modified You Only Look Once (YOLO) classifier [25] was used to demonstrate this effect in practice. The modified Tiny YOLO variant of the model presented in [13] was trained on the simulated data, and the variation in classification success for the single model analysed for test data with a range of bistatic angles.

To enable direct comparison between the imagery obtained from RaySAR and SARCASTIC, sample images have been generated with configurations matching the presented RaySAR images. The SARCASTIC simulator has been upgraded to support bistatic operation as part of this work. The target model used was the same Leopard 2A6 CAD model used for the previous RaySAR work. The model is passed through the materialise stage of the SARCASTIC toolchain to generate a Delaunay triangulation of the mesh with an appropriate surface roughness for the material. In this case, the target has been assumed to be made from a homogenous metal, for which SARCASTIC's default properties have been used.

Template compensated phase history data files are generated using the cphdShell utility. The key parameters used for this work are given in Table I. It should be noted that the aperture covers an angular range of $\pm 4.115^{\circ}$ as measured from the centre of the synthetic aperture, so it is acceptable to make the small angle approximation.

\section{RESULTS}

Figure 3 shows a comparison of simulated images containing a T72B main battle tank, L33 self-propelled gun and Leopard 2A6 for both monostatic and bistatic geometries. As explained in Subsection II-C, the monostatic geometries 


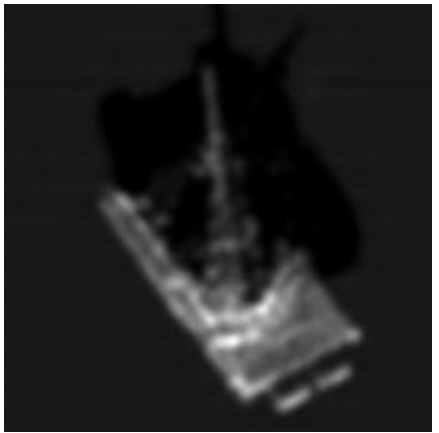

(a) T72 B, monostatic

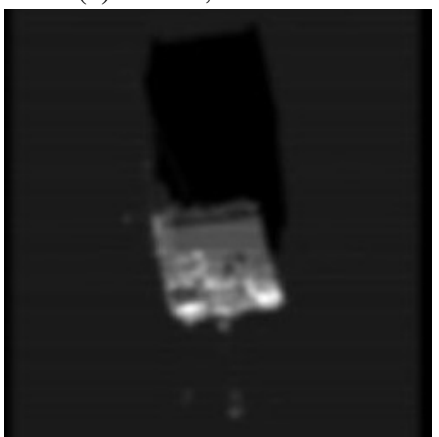

(c) L33, monostatic

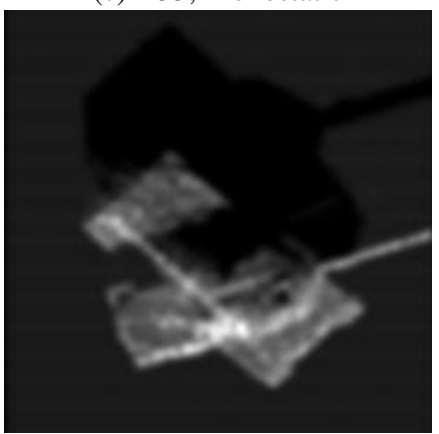

(e) Leopard 2A6, monostatic

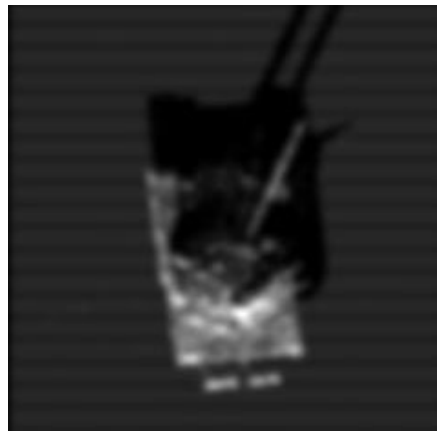

(b) $\mathrm{T} 72 \mathrm{~B}, \beta=20^{\circ}$

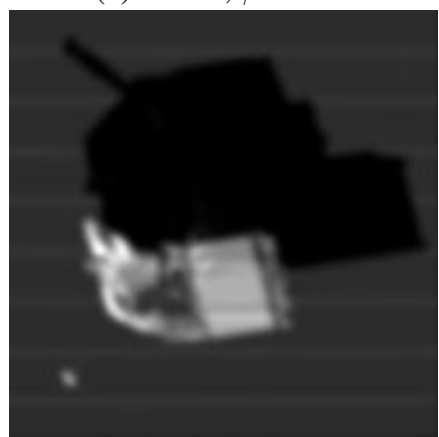

(d) $\mathrm{L} 33, \beta=90^{\circ}$

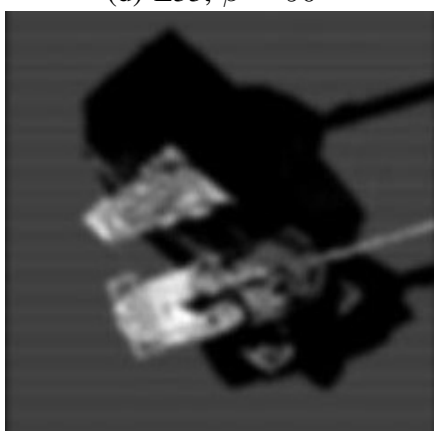

(f) Leopard 2A6, $\beta=135^{\circ}$
Fig. 3: Example images produced by the simulation pipeline presented previously in [13]

only exhibit shadowing in the range direction. For small bistatic angles such as that demonstrated in Figure $3 b$, the reconstruction and illumination shadows display high crosscorrelation.

The benefits of a bistatic geometry are clearly demonstrated by comparing Figure 3c and Figure 3d. Figure 3d was simulated with the same pose and transmitter position as Figure $3 \mathrm{c}$, but with the receiver relocated to form a bistatic angle of $90^{\circ}$. The illumination shadow in Figure $3 \mathrm{~d}$ corresponds to the reconstruction shadow in Figure $3 \mathrm{~d}$. The reconstruction shadow in the bistatic case, however, clearly displays the barrel of the $155 \mathrm{~mm}$ howitzer, which is almost invisible in the monostatic case due to the illumination incidence angle. The bistatic image also shows a strong scattering event at the muzzle brake.

It should also be noted that the length of the vehicle can

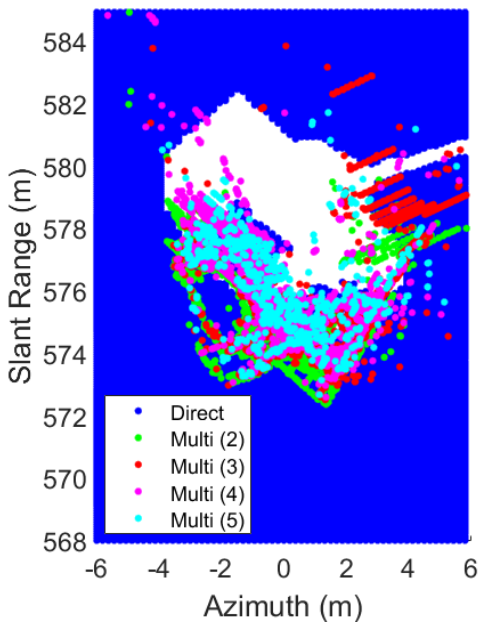

(a) Equivalent monostatic configuration (w.r.t. receiver)

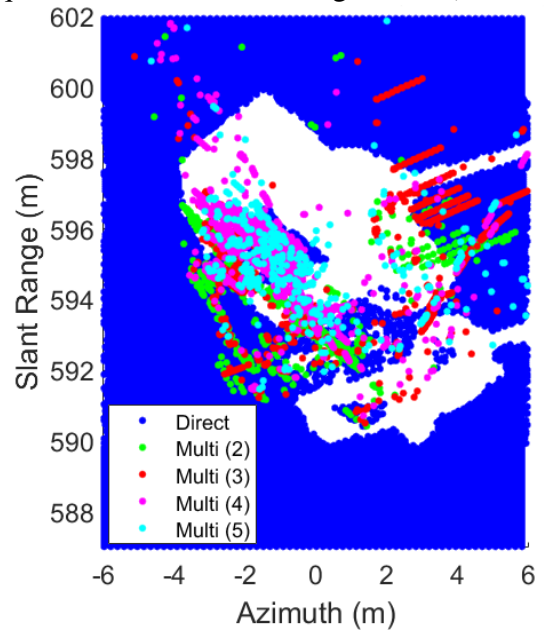

(b) Bistatic angle of $135^{\circ}$

Fig. 4: Multibounce maps showing the number of scattering events responsible for each contribution in the final image

be more accurately estimated from Figure $3 d$ as the extent of layover in the azimuth direction is limited by the geometry of the aperture. If the small angle approximation holds, then it can be assumed that azimuth layover does not occur. Fusing the azimuth and shadow measurements from the mono and bistatic images would therefore permit the derivation of the vehicle's dimensions in all three spatial axes, which can be used as features for a template-based match. Figure 4 shows a mapping of all target returns contributing to the final images in Figure $3 \mathrm{e}$ and Figure $3 \mathrm{f}$. These diagrams were obtained using the analysis tools bundled into RaySAR. The shadow regions causing occlusion of the target outline can clearly be seen; these are thought to contribute significantly to classifier performance degradation at high bistatic angles (see Subsection IV-B) as they interrupt the outline of the vehicle and obscure several prominent features on the hull. This is of particular concern for vehicles with turrets or other movable elements such as cranes or booms.

It should also be noted that there is a consistent layover 


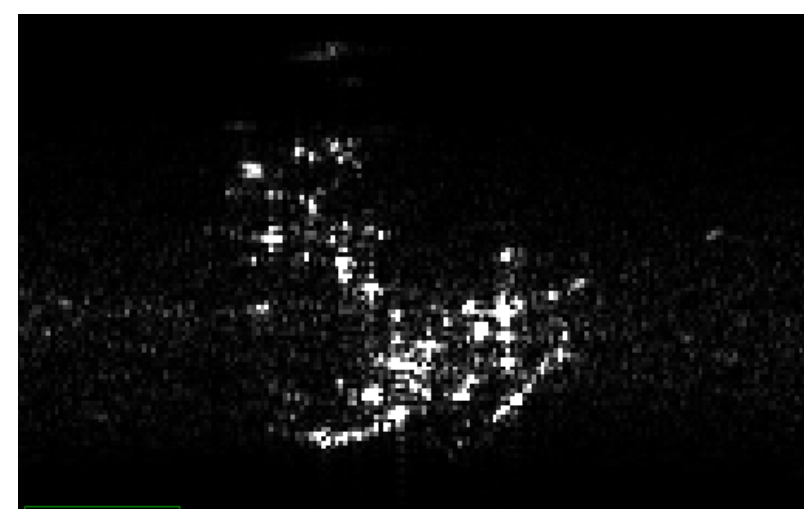

(a) Monostatic equivalent to Figure 3e)

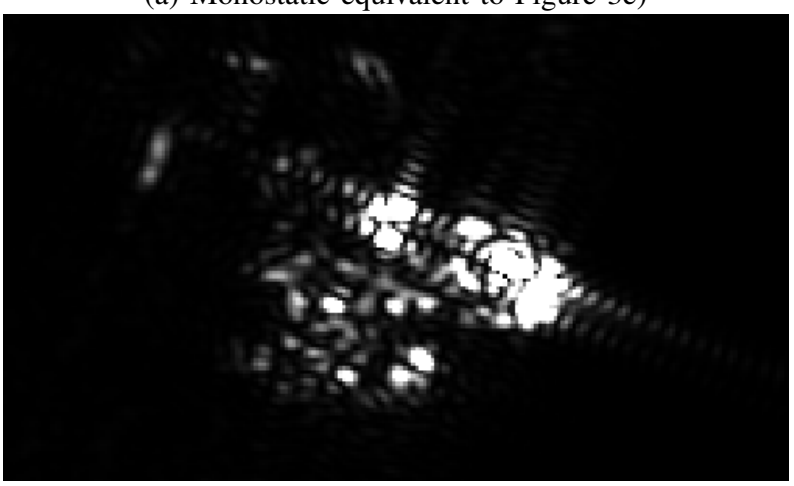

(b) Bistatic angle of $135^{\circ}$ (equivalent to Figure 3f)

Fig. 5: Simulated images of the Leopard 2A6 from the modified SARCASTIC simulator

artefact in both images where a section of the ground plane appears within the reconstruction shadow at a point where it would intersect the hull outline. The return is a direct path signal passing below the turret overhang. This has not been investigated in depth yet, but may have interesting repercussions for image segmentation approaches which rely on texture analysis to separate targets of interest from terrain.

A large reduction in multipath contributions around the turret region can be observed in the bistatic case compared to the monostatic case. The relevant area is 591 to $594 \mathrm{~m}$ slant range, -2 to $2 \mathrm{~m}$ azimuth in Figure $4 \mathrm{~b}$. Operating a bistatic configuration in the forward scatter region is likely to require significant changes to the choice of features due to the illumination shadow causing significant occlusion of parts of the target in range. However, the return from the top surfaces of the vehicle is dominated by the direct path signal, which makes feature extraction from these regions much simpler.

\section{A. SARCASTIC validation}

Figure 5 shows the matched detected imagery from SARCASTIC for the RaySAR results shown in Figure $3 \mathrm{e}$ and Figure 3f, including the first published bistatic result from SARCASTIC. These experiments were performed on the ATHENS GPU server in UCL's Electronic and Electrical Engineering department. This provides four Tesla V100 GPUs with $32 \mathrm{~GB}$ of memory each, along with four Intel Xeon Gold

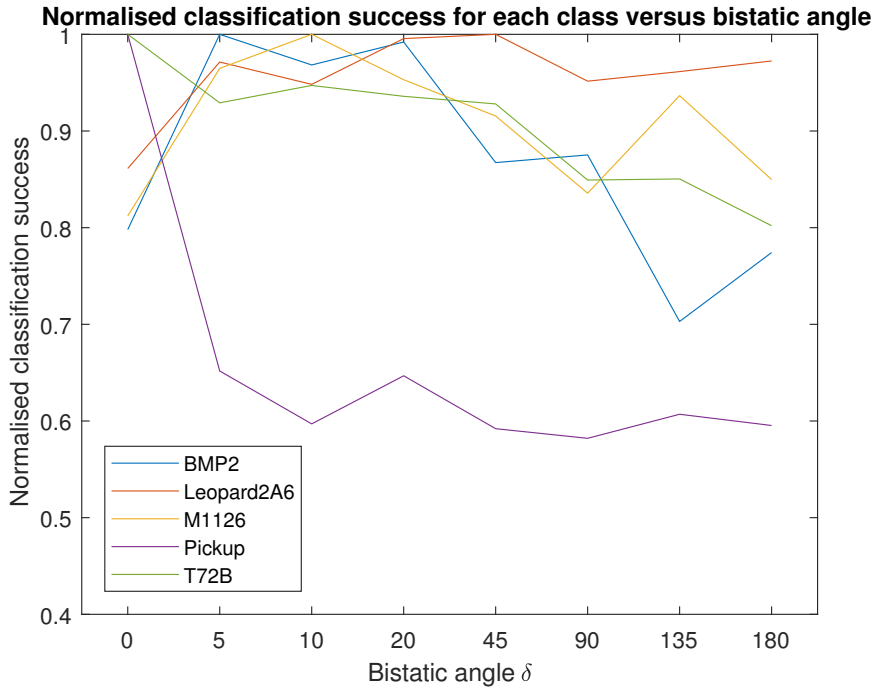

Fig. 6: Normalised classification accuracy as a function of bistatic angle for a Tiny-YOLO based classifier

6126 CPUs (48 cores, 96 threads) and $250 \mathrm{~GB}$ of RAM. The total simulation time for each image once the model was prepared was approximately 30 minutes; this equates to about $700 \mathrm{~ms}$ per pulse.

Visual comparison of the outputs of both simulators reveals common features, such as the multipath signature from the road wheels appearing inside the hull outline for the monostatic case due to layover. The predicted shadowing is present in both SARCASTIC images, and multipath artefacts are observed in many of the locations predicted by Figure 4.

\section{B. Classification success vs. bistatic angle}

The classifier described in Section III (Tiny YOLO) was trained for 15 epochs; the first 10 epochs were completed with a learning rate of 0.1 , and the last 5 used a learning rate of $10^{-3}$. K-fold cross-validation was used to provide a validation set during training with $K=10$ and stochastic gradient descent was implemented as the optimiser. Non-maximal suppression for classification was performed by selecting the highest confidence score across all predicted bounding boxes in the final layer.

Figure 6 illustrates the variance in classification success on the selected test set for the trialled Tiny YOLO classifier as the bistatic angle of the collection geometry was varied. The normalisation factor for each class was the highest achieved classification accuracy across all offsets. A number of interesting trends can be observed in this data. The tank classes (Leopard 2A6 and T72B) demonstrate a sharp drop in accuracy from $\beta=45^{\circ}$ to $\beta=90^{\circ}$; the M1126 target also demonstrated a similar characteristic. It is hypothesised that the orthogonal shadows introduced to the image when the target is illuminated at $\beta=90^{\circ}$ obscure features which have been learnt from the training data with smaller bistatic angles, leading to poor classifier performance. 


\section{CONCLUSIONS AND FUTURE WORK}

In this paper, it has been demonstrated that bistatic collection geometries introduce complex artefacts and shadowing in SAR imagery, particularly when a target introduces overhangs and abrupt changes in elevation which are vulnerable to layover. A large quantity of simulated imagery has been produced to support research into these effects and analysis of a small number of examples has been presented. A direct comparison between the fast RaySAR and high-fidelity SARCASTIC simulators has been made to demonstrate their relative strengths. A Tiny YOLO classifier was trained on the dataset, and its performance was found to vary significantly depending on the bistatic angle used to generate the input image. This validates the assertion that ATR methodologies for bistatic systems can neither ignore the effects of a change in collection geometry nor assume that the same approaches used for monostatic scenarios will function as expected.

The hybrid pipeline mentioned in Section III and the upgraded SARCASTIC simulator will be exploited to support a simulation campaign covering more targets, along with realistic terrain models. Building a comprehensive dataset containing many targets of interest over a large parameter space will enable further investigation into the nature of unusual artefacts in bistatic SAR imagery, the frequency with which they occur, and methods for mitigating or exploiting them in an operational context. A large subset of this data will be openly released to enable wider research into the problem of bistatic SAR ATR. Using the SARCASTIC simulator will also allow the full complex image to be investigated; exploiting the phase data will give much greater insight into the structure of the target response. From the observation that the phase of noise contributions will be largely uncorrelated whereas multipath artefacts arising from the target will be correlated to the physical geometry, it follows that this could be used to identify multipath artefacts in the shadow regions. This could also be exploited to improve shadow segmentation, along with texture analysis.

\section{ACKNOWLEDGEMENTS}

This work was supported by funding from Dstl and EPSRC Grant EP/R513143/1. Bright Spark imagery courtesy of Thales,UK and Dstl.

\section{REFERENCES}

[1] C. W. Sherwin, J. Ruina, and R. Rawcliffe, "Some early developments in synthetic aperture radar systems," IRE Transactions on Military Electronics, vol. 1051, no. 2, pp. 111-115, 1962.

[2] The Air Force Moving and Stationary Target Recognition Database. [Online]. Available: https://www.sdms.afrl.af.mil/datasets/mstar/

[3] E. E. Laubie, B. D. Rigling, and R. P. Penno, "Bistatic sar image registration accuracy," in 2015 IEEE Radar Conference, May 2015, pp. 0742-0746.

[4] J. B. Keller, "Geometrical theory of diffraction," J. Opt. Soc. Am., vol. 52, no. 2, pp. 116-130, Feb 1962. [Online]. Available: http://www.osapublishing.org/abstract.cfm?URI=josa-52-2-116

[5] B. D. Rigling and R. L. Moses, "GTD-based scattering models for bistatic SAR," in Algorithms for Synthetic Aperture Radar Imagery XI, E. G. Zelnio and F. D. Garber, Eds., vol. 5427, International Society for Optics and Photonics. SPIE, 2004, pp. 208 - 219. [Online]. Available: https://doi.org/10.1117/12.541555
[6] F. Comblet, A. Khenchaf, A. Baussard, and F. Pellen, "Bistatic synthetic aperture radar imaging: Theory, simulations, and validations," IEEE Transactions on Antennas and Propagation, vol. 54, no. 11, pp. 35293540, Nov 2006.

[7] J. A. Jackson, B. D. Rigling, and R. L. Moses, "Parametric scattering models for bistatic synthetic aperture radar," in 2008 IEEE Radar Conference, May 2008, pp. 1-5.

[8] - "Canonical scattering feature models for 3D and bistatic SAR," IEEE Transactions on Aerospace and Electronic Systems, vol. 46, no. 2, pp. 525-541, April 2010.

[9] M. Hazlett, D. J. Andersh, S. W. Lee, H. Ling, and C. L. Yu, "XPATCH: a high-frequency electromagnetic scattering prediction code using shooting and bouncing rays," in Targets and Backgrounds: Characterization and Representation, W. R. Watkins and D. Clement, Eds., vol. 2469, International Society for Optics and Photonics. SPIE, 1995, pp. 266 - 275. [Online]. Available: https://doi.org/10.1117/12. 210627

[10] D. Andersh, J. Moore, S. Kosanovich, D. Kapp, R. Bhalla, R. Kipp, T. Courtney, A. Nolan, F. German, J. Cook, and J. Hughes, "Xpatch 4: the next generation in high frequency electromagnetic modeling and simulation software," in Record of the IEEE 2000 International Radar Conference [Cat. No. O0CH37037], May 2000, pp. 844-849.

[11] S. Auer, R. Bamler, and P. Reinartz, "Raysar - 3D SAR simulator: Now open source," in 2016 IEEE International Geoscience and Remote Sensing Symposium (IGARSS), July 2016, pp. 6730-6733.

[12] S. Auer, S. Hinz, and R. Bamler, "Ray-tracing simulation techniques for understanding high-resolution SAR images," IEEE Transactions on Geoscience and Remote Sensing, vol. 48, no. 3, pp. 1445-1456, 2010.

[13] M. Woollard, A. Bannon, M. Ritchie, and H. Griffiths, "Synthetic aperture radar automatic target classification processing concept," Electronics Letters, 2019.

[14] D. Muff, M. Stevens, D. Blacknell, M. Nottingham, C. Stevenson, and H. Griffiths, "Detecting vibrating targets in fine resolution SAR imagery," in EUSAR 2018; 12th European Conference on Synthetic Aperture Radar, June 2018, pp. 1-6.

[15] D. G. Muff, "Electromagnetic ray-tracing for the investigation of multipath and vibration signatures in radar imagery," Ph.D. dissertation, UCL (University College London), 2018.

[16] S. Papson and R. M. Narayanan, "Classification via the shadow region in SAR imagery," IEEE Transactions on Aerospace and Electronic Systems, vol. 48, no. 2, pp. 969-980, April 2012.

[17] M. Heiligers and A. Huizing, "On the importance of visual explanation and segmentation for SAR ATR using deep learning," in 2018 IEEE Radar Conference (RadarConf18), April 2018, pp. 0394-0399.

[18] R. R. Selvaraju, M. Cogswell, A. Das, R. Vedantam, D. Parikh, and D. Batra, "Grad-cam: Visual explanations from deep networks via gradient-based localization," in Proceedings of the IEEE International Conference on Computer Vision, 2017, pp. 618-626.

[19] K. Simonyan, A. Vedaldi, and A. Zisserman, "Deep inside convolutional networks: Visualising image classification models and saliency maps," arXiv preprint arXiv:1312.6034, 2013.

[20] R. D. Chaney, A. S. Willsky, and L. M. Novak, "Coherent aspectdependent SAR image formation," in Algorithms for Synthetic Aperture Radar Imagery, vol. 2230. International Society for Optics and Photonics, 1994, pp. 256-274.

[21] X. Mao, Y. D. Zhang, and M. G. Amin, "Low-complexity sparse reconstruction for high-resolution multi-static passive SAR imaging," EURASIP Journal on Advances in Signal Processing, vol. 2014, no. 1, p. $104,2014$.

[22] D. Malmgren-Hansen and M. Nobel-Jørgensen, "Convolutional neural networks for SAR image segmentation," in 2015 IEEE International Symposium on Signal Processing and Information Technology (ISSPIT), Dec 2015, pp. 231-236.

[23] C. Belloni, "Deep learning and feature-based classification techniques for radar imagery," Ph.D. dissertation, Cranfield University, UK, 2019.

[24] M. Jahangir, D. Blacknell, C. Moate, and R. Hill, "Extracting information from shadows in SAR imagery," in 2007 International Conference on Machine Vision. IEEE, 2007, pp. 107-112.

[25] J. Redmon and A. Farhadi, "YOLO9000: better, faster, stronger," arXiv preprint, 2017. 